Cereal Research Communications 43(2), pp. 284-294 (2015)

DOI: $10.1556 / C R C .2014 .0041$

First published online 4 February, 2015

\title{
Effects of Allelic Variation in Glutenin Subunits and Gliadins on Baking-quality in Near-isogenic Lines of Common Wheat cv. Longmai 19
}

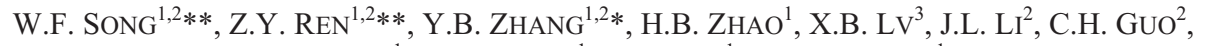 \\ Q.J. SONG ${ }^{1}$, C.L. ZHANG ${ }^{1}$, W.L. XIN ${ }^{1}$ and Z.M. XIAO ${ }^{1}$ \\ ${ }^{1}$ Crop Breeding Institute, Heilongjiang Academy of Agricultural Sciences, Harbin 150086, PR China \\ ${ }^{2}$ College of Life Science and Technology, Harbin Normal University, Harbin 150025, PR China \\ ${ }^{3}$ Biotechnology Research Institute, Heilongjiang Academy of Agricultural Sciences, Harbin 150086, PR China \\ (Received 6 March 2014; Accepted 16 June 2014; \\ Communicated by F. Békés)
}

Two lines, L-19-613 and L-19-626, were produced from the common wheat cultivar Longmai 19 (L-19) by six consecutive backcrosses using biochemical marker-assisted selection. L-19 (Glu-Dla, Glu-A3c/Gli-A1?; Gli-A1? is a gene coding for unnamed gliadin) and L-19-613 (Glu-D1d, Glu-A3c/Gli-A1?) formed a set of near-isogenic lines (NILs) for HMW-GS, while L-19-613 and L-19-626 (Glu-Dld, Glu-A3e/Gli-Alm) constituted another set of NILs for the LMW-GS/gliadins. The three L-19 NILs were grown in the wheat breeding nursery in 2007 and 2008. The field experiments were designed using the three-column contrast arrangement method with four replicates. The three lines were ranked as follows for measurements of gluten strength, which was determined by the gluten index, Zeleny sedimentation, the stability and breakdown time of the farinogram, the maximum resistance and area of the extensogram, and the P and W values of the alveogram: L-19-613 > L-19-626 > L-19. The parameters listed above were significantly different between lines at the 0.05 or 0.01 level. The Glu-D1 and Glu-A3/Gli-A1 loci had additive effects on the gluten index, Zeleny sedimentation, stability, breakdown time, maximum resistance, area, $\mathrm{P}$ and $\mathrm{W}$ values. Although genetic variation at the $G l u-A 3 / G l i-A 1$ locus had a great influence on wheat quality, the genetic difference between Glu-DId and Glu-DIa at the Glu-D1 locus was much larger than that of Glu-A3c/Gli-A1? and Glu-A3e/Gli-A1m at the Glu-A3/Gli-A1 locus. Glu-D1d had negative effects on the extensibility and the L value compared with Glu-Dla. In contrast, Glu-A3c/ Gli-A1? had a positive effect on these traits compared with Glu-A3e/Gli-Alm.

Keywords: common wheat, quality, HMW-GS, LMW-GS, gliadin block, NILs

\footnotetext{
* Corresponding author; E-mail: yanbin_zhang@163.com

** The first two authors contributed equally.
} 


\section{Introduction}

Wheat baking quality mainly depends on two types of storage proteins: glutenins and gliadins. Glutenins are further divided into two groups according to their molecular weights and mobility in sodium dodecyl sulphate-polyacrylamide gel electrophoresis (SDS-PAGE): the high-molecular-weight glutenin subunit (HMW-GS) and the low-molecular-weight glutenin subunit (LMW-GS) (Bietz and Wall 1972). HMW-GS and LMW-GS are encoded by genes at the Glu-1 and Glu-3 loci, which are located on the long and short arms of homoeologous group 1 chromosomes, respectively (Payne 1987; Gianibelli et al. 2001)). Gliadins are divided into four subgroups based on their mobility in acid polyacrylamide gel electrophoresis (A-PAGE): $\alpha$ (the fastest), $\beta, \gamma$, and $\omega$ (the slowest) (Bushuk and Zillman 1978; Gianibelli et al. 2001). Genes encoding gliadin components are located at the Gli-1 (Gli-A1, Gli-B1, and Gli-D1) and Gli-2 (Gli-A2, Gli-B2, and Gli-D2) loci on the short arms of homologous group 1 and 6 chromosomes (Payne 1987; Gianibelli et al. 2001). Some studies indicated that some gliadin bands are tightly linked and inherited together; these components are called gliadin blocks (Sozinov and Poperelya 1970; Sozinov and Poperelya 1980; Sozinov and Poperelya 1982). Alleles of 111 gliadins were identified and named by Metakovsky (1991). At the same time, a list of standard cultivars was also defined, which laid a solid foundation for the study and utilisation of gliadin.

HMW-GS can be easily distinguished by 1-D SDS-PAGE (Gupta and Shepherd 1990; Gianibelli et al. 2001). The effect of HMW-GS on baking quality mainly depends on the composition of the HMW-GS and the quantity of each subunit (Sontag-Strohm et al. 1996; Ragupathy et al. 2008; Jin et al. 2012). The results of these HMW-GS studies were widely used in wheat quality breeding for determining which parents to cross and for selection of progeny (Redaelli et al. 1997; Fang et al. 2009; Rasheed et al. 2012). The structure of LMW-GS is similar to that of HMW-GS, and the composition of these molecules is also strongly related to wheat baking quality (Payne 1987; Gupta and Shepherd 1990; Sontag-Strohm et al. 1996; Redaelli et al. 1997; Gianibelli et al. 2001). However, the LMW-GS are difficult to distinguish by 1-D SDS-PAGE, mainly because of their overlapping and tight genetic linkage with the gliadins (D'Ovidio and Masci 2004; Zhang et al. 2012). Thus, compared with HMW-GS, much less is known about the effects of LMW-GS (Redaelli et al. 1995; Gianibelli et al. 2001). Based on sequence polymorphisms among LMW-GS genes, functional markers were developed and used for marker-assisted selection in wheat breeding (Wang et al. 2010; Liu et al. 2012). According to the reported by Liu et al. (2010), functional markers developed by Wang et al. (2010) are the simplest, most accurate, lowest cost method for identification of Glu-A3 alleles, compared to the SDS-PAGE, two-dimensional gel electrophoresis (2-DE, IEF $\times$ SDS-PAGE), matrix-assisted laser desorption/ionization time-of-flight mass spectrometry (MALDI-TOF-MS). Based on conserved structure and length polymorphisms among LMW-GS genes, a new marker system was developed for identifying LMW-GS genes (Zhang et al. 2011). However, further work is necessary to characterize LMW-GS genes in hundreds of wheat varieties with this new marker system (Zhang et al. 2011). 
Here, we carried out six consecutive backcrosses with Longmai 19 (HMW-GS composition $\left.2^{*}, 7^{*+9}, 2+12\right)$ as the recurrent parent and the Canadian wheat variety Marquis (HMW-GS composition $1,7^{*+9}, 5+10$ ) as the donor of subunits $5+10$ in the backcrosses. The sister lines L-19-613 and L-19-626 were formed from this process. HMW-GS (encoded by Glu-D1) and LMW-GS/gliadin (encoded by Glu-A3/Gli-A1) were identified by SDS-PAGE, A-PAGE (Jiang et al. 2011), and functional markers for the Glu-A3 locus (Song et al. 2012). The only difference between L-19 and L-19-613 was the Glu-D1 locus; thus, these lines formed a pair of Glu-Dla/Glu-D1d near-isogenic lines (NILs). Similarly, L-19-626 (Glu-A3e/Gli-Alm) and L-19-613 (Glu-A3c/Gli-A1?; Gli-A1? is a gene coding for unnamed gliadin) formed a pair of Glu-A3/Gli-A1 NILs. L-19-626 had the closely linked Glu-A3e/Gli-Alm gene from its 5+10 subunit donor, Marquis, while L-19 and L-19-613 had the closely linked Glu-A3c/Gli-A1? gene. The aims of this study were to precisely evaluate the genetic effects of different subunits on the quality of NILs of the wheat cultivar Longmai 19 and to provide useful information for breeding programmes aimed at enhancing wheat quality.

\section{Materials and Methods}

\section{Plant materials and field experiment design}

The HMW-GS composition of L-19 was $2^{*}, 7^{*+9}$, and $2+12$, and the alleles at the Glu-A3/Gli-A1 locus were Glu-A3c/Gli-A1?. The HMW-GS composition of L-19-613 was 2*, $7^{*}+9$, and 5+10, with Glu-A3c/Gli-A1? at the Glu-A3/Gli-Al locus. L-19-626 had a HMW-GS composition of $2^{*}, 7^{*+9}, 5+10$, with alleles Glu-A3e/Gli-Alm at the Glu-A3/Gli-A1 locus. Except for the loci mentioned above, there were no differences in glutenins and gliadins among L-19, L-19-613, and L-19-626. The field experiments were designed using the three-column contrast arrangement method with four replicates, and the experiments were performed in 2007 and 2008 at the Heilongjiang Academy of Agricultural Sciences, Harbin, China.

\section{Electrophoresis}

Glutenins were fractionated by SDS-PAGE as described by Ji et al. (2008) except that the separating gel was changed to a concentration of $12 \%$. Gliadins were fractionated by A-PAGE as described by Zhang et al. (1997) with the exception that both the separating gel and the stacking gel were changed to a concentration of $6 \%$. The HMW-GS were named following the nomenclature of Payne and Lawrence (1983), and the LMW-GS were named following Gupta and Shepherd (1990). The gliadins were classified using the nomenclature of Metakovsky (1991).

\section{Quality analysis}

A Quadrumat ${ }^{\circledR}$ Senior mill (Brabender Co., Germany) was used to produce wheat flour according to the standard method of the American Association of Cereal Chemists 26-20 (AACC 26-20). The flour protein content was determined using a DA7200 Diode Array 
Analyzer (Perten Co., Sweden). The wet and dry gluten content as well as the gluten index were obtained using a Glutomatic 2200 (Glutomatic System, Perten Co.) according to the National Standard of China 14608-93 (GB/T 14608-93). The Zeleny sedimentation value was recorded using a shaker (Brabender Co.), according to AACC 56-61. Dough rheological characters were determined using a Micro-Farinograph (Brabender Co.), an Extensograph $^{\circledR}$ (Brabender Co.), and an MA82 Chopin Alveograph (Tripette et Renaud, France) according to AACC54-21, AACC54-10, and ISO 5530-4, respectively.

\section{Results}

\section{SDS-PAGE and A-PAGE analysis}

The glutenins and gliadins of L-19 NILs were analysed by SDS-PAGE and A-PAGE. The only difference between L-19 and L-19-613 was HMW-GS (encoded by Glu-D1), and the only difference between L-19-626 and L-19-613 was LMW-GS/gliadins (encoded by Glu-A3/Gli-A1) (Figs S1 and S2*). An LMW-GS band identified in both L-19 and L-19-613 by SDS-PAGE, but not observed in L-19-626 or Marquis, was termed the L-19-613-specific band (Fig. S1); the mobility of this band was the same as that of the LMW-GS Glu-A3c. Similarly, an L-19-626-specific LMW-GS band was identified on the L-19-626 and Marquis glutenin SDS-PAGE electrophoretogram but was not found in L-19 or L-19-613 (Fig. S1).

\section{Protein, gluten and sedimentation values}

The greatest difference in average flour protein content among the three Longmai 19 NILs from the two study years was $0.4 \%$ (Table S1). The maximum difference in average dry gluten content between L-19-626 and L-19-613 was 1\% in 2007 and was $0.3 \%$ in 2008. That is, the NILs of Glu-A3e/Gli-Alm had a slightly but significantly higher quantity of protein than those of Glu-A3c/Gli-A1? $(p<0.01)$.

The wet gluten content of L-19-613 was the lowest among the NILs in both study years (Table S1), with a mean content across both years that was 5\% lower than that of L-19-626 and was $3 \%$ lower than that of L-19 ( $p<0.01$ for both). The difference between NILs may be attributed to the stronger contraction force of gluten in L-19-613, which resulted in its low water retention rate.

L-19-613 had the highest gluten index, followed by L-19-626 and L-19 (Table S1). The 2-year average gluten index of L-19-613 was $18.6 \%$ and $41.3 \%$ higher than those of L-19-626 and L-19, respectively $(p<0.01)$.

L-19-613 also had the highest 2-year average Zeleny sedimentation value, followed by L-19-626 and L-19 (Table S1). The 2-year average of L-19-613 was $12.7 \%(p<0.01)$ and $18.6 \%(p<0.01)$ higher than those of L-19-626 and L-19, respectively. The 2-year average of L-19-626 was also significantly higher than that of L-19 $(p<0.05)$.

\footnotetext{
* Further details about the Electronic Supplementary Material (ESM) can be found at the end of the article.
} 


\section{Farinograph parameters}

L-19-626 had the greatest 2-year average water absorption, followed by L-19 and L-19-613 (Table S1). Determining the cause of this difference will require further study. However, the differences were slight, with the greatest difference $(1.1 \%)$ between L-19-626 and L-19-613. The development time did not show consistent results in the L-19 NILs over the two years, possibly due to environmental effects. The stability and breakdown time of L-19-613 were $28.1 \%$ and $18.0 \%$ greater than those of L-19-626 and were $95.2 \%$ and $51.3 \%$ greater than those of L-19, respectively. The differences in the 2-year average stability and breakdown time among the three NILs were significant at the 0.01 level.

\section{Extensograph parameters}

L-19-613 had a greater maximum resistance and area in the extensogram, followed by L-19-626 and L-19 (Table S1). The 2-year average of maximum resistance and area of L-19-613 were $46.3 \%$ and $51.7 \%$ higher than those of L-19-626 and were $133.3 \%$ and $121.3 \%$ higher than those of L-19 (both $p<0.01$ ). The 2-year extensibility of L-19 was greater than that of L-19-613, followed by L-19-626. The differences in the 2-year average extensibility of the three NILs were all significant $(p<0.01)$. The extensibility of L-19-626 was the lowest, which indicates a negative effect of Glu-D1d and Glu-A3e/Gli-Alm.

\section{Alveograph parameters}

As illustrated in the alveogram, L-19-613 had the highest average $\mathrm{P}$ and $\mathrm{W}$ values for both years, followed by L-19-626 and L-19 (Table S1). The 2-year average P and W values of L-19-613 were 7.4\% $(p=0.05)$ and 18.2\% $(p=0.01)$ higher than those of L-19-626 and were $21.5 \%(p=0.01)$ and $37.4 \%(p=0.01)$ higher than those of L-19, respectively. In 2008, L-19 had the greatest L value, followed by L-19-613 and L-19-626; this order was the same as the extensibility measured using the extensograph. However, in 2007, L-19-613 had the greatest L value, followed by L-19-626 and L-19. The reason that the performance of L-19 differed so greatly over the two years is still under study. Compared with $G l u-A 3 c / G l i-A 1$ ?, the two years of data from the alveograph indicate that Glu-A3e/Gli-Alm has a negative influence on $\mathrm{P}, \mathrm{W}$, and $\mathrm{L}$ values.

\section{Discussion}

The Longmai 19 NILs provided excellent material for genetic analysis (Popineau et al. 1994; Sontag-Strohm et al. 1996; Redaelli et al. 1997; Lv et al. 2004; Zhang et al. 2008, 2010). First, the NILs have the same genetic background except for the gene under analysis. The influence of genetic background on gene analysis was therefore eliminated in the NILs. Second, the use of NILs with the same developmental stages and maturation periods can minimise the impact of environmental factors on the NILs with different subunits. 
Therefore, the character differences of the NILs reflect the genetic differences of specific genes in certain environments (Zhang et al. 2010).

The flour protein content of L-19-613, L-19-626, and L-19 in 2008 was $0.4 \%, 0.4 \%$, and $0.5 \%$ higher than in 2007 , respectively. However, dry gluten content decreased by $0.4 \%, 1.1 \%$, and $0.4 \%$, and wet gluten content decreased by $3.2 \%, 4.2 \%$, and $2.0 \%$, respectively, from 2007 to 2008 . Although the wet and dry gluten content were lower in 2008 than in 2007, other quality parameters, including the gluten index, sedimentation value, stability and breakdown time of the farinograph, maximum resistance and area of the extensograph, and W value of the alveograph, were higher in 2008. The same phenomenon was also observed in a previous study of HMW-GS NILs (Zhang et al. 2008). On the one hand, the results indicate that flour protein contains both gluten and non-gluten proteins; thus, the flour protein content was not always directly proportional to the gluten protein content. On the other hand, various factors influence wheat quality and can cause complex changes. Even in the same cultivar, high protein content is not necessarily correlated with high gluten content, and high gluten content does not indicate high quality. The finding that non-gluten protein content increased while gluten protein content decreased in all NILs suggests that all NILs shared the same genetic background and that their response to environmental change also remained consistent.

Some studies indicate that variations at the Glu-D1 and Glu-A3/Gli-Al loci have little influence on the quantity of gluten but have a greater influence on the quality of gluten (Sontag-Strohm et al. 1996; Redaelli et al. 1997; Lv et al. 2004; Zhang et al. 2008, 2010); these findings are in accord with our results. In this study, the NILs with the gene Glu-A3e had slightly increased protein and dry gluten content. However, these increases were not significant. The differences in gluten quantity (protein and dry gluten content) among the L-19 NILs were small. Therefore, the quality differences among the L-19 NILs were caused by protein quality rather than quantity.

Following the method proposed by Ji et al. (2008), 7.5\% isopropanol and $0.3 \mathrm{~mol} / \mathrm{L} \mathrm{NaI}$ were employed to remove prolamin and other monomeric proteins. After extracting all the wheat glutenin subunits from L-19-626, a key protein, termed the L-19-626-specific band, was identified using SDS-PAGE. Bands with same mobility were also found in seven wheat varieties, including Marquis (Canada), Neepawa (Canada), Klein Martillo (Argentina), Halberd (Australia), Magali Blondeau (France), Aoba-komugi (Japan), and Splendor (USA) (data not shown), which possessed the LMW-GS alleles Glu-A3e at Glu-A3 locus identified by SDS-PAGE, 2-DE, MALDI-TOF-MS and functional markers, according to the results reported by Liu et al. (2010). In segregating populations derived from a cross of L-19 and L-19-626, we found that the lines with an L-19-626-specific band identified by SDS-PAGE contained Gli-A1m and Glu-A3e, as identified by A-PAGE and the functional markers for Glu-A3e, and the lines with an L-19-613-specific band identified

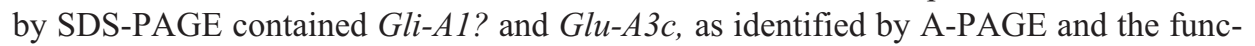
tional markers for Glu-A3c (Song et al. 2012). Gupta et al. (1990) reported that the Glu-A3e subunit is not expressed; thus, the band could have been a closely linked protein marker of Glu-A3e if it was not the subunit encoded by Glu-A3e. The study of Liu et al. 
(2010) revealed that Glu-A3e possesses a special peak in MALDI-TOF-MS. The studies on the nature of the L-19-D626-specific band are in progress.

The values of most wheat quality parameters related to strong gluten were greatest in L-19-D613, followed by L-19-D626 and L-19. Based on the percentage increase in the different quality parameters, variations in the two loci had the greatest effect on maximum resistance, followed by the area of the extensogram, stability, breakdown time, and the gluten index. The same genetic variation had different effects on other quality parameters, suggesting that most quality parameters are correlative, yet relatively independent (Zhang et al. 2008).

Some studies have suggested that the $\mathrm{W}$ and $\mathrm{P}$ values of the alveograph are closely correlated with the area and maximum resistance of the extensograph; this relationship was significant at the 0.01 level. Typically, data from the alveograph and the extensograph are highly consistent (Wei 1998; Zhang et al. 2006; Li 2009). Although the three NILs were weak-gluten cultivars, as indicated by the maximum resistance measured by the extensograph, the $\mathrm{P}$ value generated from the alveograph suggested that the three NILs were either strong- (2007) or medium-gluten varieties (2008). Thus, the P value was not consistent with maximum resistance, which is consistent with the results of our previous study (Ding et al. 2012).

The effects of the alleles Glu-1 and Glu-A3/Gli-A1 are largely additive (Gupta et al. 1989; Sontag-Strohm et al. 1996; Redaelli et al. 1997). In the present study, the effects of the alleles Glu-D1, encoding HMW-GS, and Glu-A3/Gli-A1, encoding LMW-GS and gliadins, on gluten index, sedimentation value, stability of the farinograph, and the maximum resistance and area of the extensograph were largely additive.

Gupta et al. (1989) noted that "The effect of the LMW glutenin allele Glu-A3m on both dough resistance and dough extensibility was relatively larger than that of the HMW glutenin allele $G l u-A 1 b$ at both sites". In this study, although genetic variation at the Glu-A3/Gli-A1 locus had a great influence on wheat quality, the genetic difference between the $d$ and $a$ alleles of the Glu-Dl locus was much greater than that of the $\mathrm{c} /$ ? and $\mathrm{e} / \mathrm{m}$ alleles at the Glu-A3/Gli-Al locus. Based on the 2-year average, the contribution of the Glu-Dl locus to differences in maximum resistance and area of the extensograph, stability and breakdown time of the farinograph, and gluten index was twice as great as that of the Glu-A3/Gli-A1 locus.

Zhang et al. (2012) reported that $G l u-A 3 e$ produced the lowest quality parameters among five $G l u-A 3$ alleles (including $G l u-A 3 b, G l u-A 3 c, G l u-A 3 d$, Glu-A3f). The results showed that Zeleny sedimentation value, stability, the maximum resistance, area and extensibility of GluA-3c were $15.7 \%, 54.3 \%, 74.2 \%, 86.3 \%$ and $13.7 \%$ higher than those of GluA-3e in the genetic background of Aroona, respectively. In this study, the results showed that the 2-year average of Zeleny sedimentation value, stability, the maximum resistance, area and extensibility of GluA-3c were $12.7 \%, 28.1 \%, 46.3 \%, 51.7 \%$ and $4.9 \%$ higher than those of GluA-3e in the genetic background of L-19 with 5+10 subunit, respectively. In GluA-3 locus, GluA-3c showed better quality parameters than GluA-3e in diverse genetic background. 
In this study, compared with the $5+10$ subunit, the $2+12$ subunit had a negative influence on the maximum resistance and area measured by the extensograph and the $\mathrm{P}$ and $\mathrm{W}$ values measured by the alveograph, but had a positive influence on extensibility and $\mathrm{L}$ value, which is consistent with the results of previous studies (Redaelli et al. 1997; $\mathrm{Lv}$ et al. 2004). However, Gli-Alm/Glu-A3e had a negative influence on extensibility and L value compared with $G l i-A 1$ ?/Glu-A3c. Therefore, Gli-A1m/Glu-A3e appears to be an inferior gene, which is undesirable for wheat breeding for either strong or medium gluten (Gupta et al. 1989; Zhang et al. 2012).

Because of the close linkage between Gli-Alm alleles and Glu-A3e alleles, it is easier to identify gliadin Gli-Alm using A-PAGE than to determine LMW-GS Glu-A3e using SDS-PAGE. Identification of gliadins is simpler, less expensive, and easily adoptable to wheat breeding programmes (Singh and Shepherd 1988; Redaelli et al. 1995; Melnikova et al. 2012).

The suggestion that the influence of variation at the Glu-A3/Gli-A1 locus on wheat quality (i.e., the quality difference between L-19-613 and L-19-626) is caused by differences in LMW-GS or gliadin is still controversial (Payne et al. 1984; Gupta et al. 1989; Nieto-Taladriz et al. 1994; Khatkar et al. 2002a, b). Some studies have suggested that gliadin alleles at the Gli-1 locus influence wheat gluten strength (Nieto-Taladriz et al. 1994; Khatkar et al. 2002a, b), while others attribute the influence of Gli-1 to the close linkage with Glu-3 alleles (Payne et al. 1984; Gupta et al. 1989). The results of the present study on the structure of glutenin and gliadin suggest that the quality difference between NILs L-19-613 and L-19-626 was mainly caused by LMW-GS.

\section{Acknowledgements}

This work was supported by National Natural Science Foundation of China (30871525), National Key Technology R\&D program (2011BAD35B03), Modern Agricultural Technical System of China (CARS-3-1-6). We are very grateful to Dr. Xia Xianchun and Liu Li, Institute of Crop Sciences, Chinese Academy of Agricultural Sciences, for their kind help on identifing of the LMW-GS. We would like to give our thanks to Dr Sun Lianfa, Institute of Crop Sciences, Heilongjiang Academy of Agricultural Sciences, for his kind help on the English editing of this article.

\section{References}

Bietz, J.A., Wall, J.S. 1972. Wheat gluten subunits: Molecular weights determined by sodium sulfate-polyacrylamide gel electrophoresis. Cereal Chem. 49:416-430.

Bushuk, W., Zillman, R.R. 1978. Wheat cultivar identification by gliadin electrophoregrams. I. Apparatus, method and nomenclature. Can. J. Plant Sci. 58:505-515.

Ding, Q., Zhang, Y.B., Li, J.L., Zhang, X.X., Zhao, H.B., Song, Q.J., Zhang, C.L., Xin, W.L., Xiao, Z.M. 2012. Preliminary study on dynamic change of dough rheological properties of pre-harvest sprouting wheat. Heilongjiang Agri. Sci. 5:1-5. (in Chinese)

D’Ovidio, R., Masci, S. 2004. The low-molecular-weight glutenin subunits of wheat gluten. J. Cereal Sci. 39:321-339. 
Fang, J.Y., Liu, Y., Luo, J., Wang, Y.S., Shewry, P.R., He, G.Y. 2009. Allelic variation and genetic diversity of high molecular weight glutenin subunit in Chinese endemic wheats (Triticum aestivum L.). Euphytica 166:177-182.

Gianibelli, M.C., Larroque, O.R., MacRitchie, F., Wrigley, C.W. 2001. Biochemical, genetic, and molecular characterization of wheat endosperm protiens. American Association of Cereal Chemists. Publication no. C-2001-0926-01O. (Online review). pp. 1-20. Available: http://www.aaccnet.org/cerealchemistry/freearticle/ gianibelli.

Gupta, R.B., Singh, N.K., Shepherd, K.W. 1989. The cumulative effect of allelic variation in LMW and HMW glutenin subunits on dough properties in the progeny of two bread wheats. Theor. Appl. Genet. 77:57-64.

Gupta, R.B., Shepherd, K.W. 1990. Two-step one-dimensional SDS-PAGE analysis of LMW subunits of glutelin. Theor. Appl. Genet. 80:65-74.

Ji, J., Liu, D.C., Wang, J., Li, J.M., Zhang, A.M. 2008. A method of extraction and separation of wheat gluten. Hereditas 30:123-126. (in Chinese)

Jiang, H.H., Zhang, Y.B., Li, J.L., Ren, Z.Y., Ding, Q., Zhao, H.B., Song, Q.J., Zhang, C.L., Xin, W.L., Xiao, Z.M. 2011. Identification of gliadin block from donor variety of HMW-GS 5+10 in NILs of Longmai 19 at Glu-D1 Loci. Heilongjiang Agri. Sci. 5:1-3. (in Chinese)

Jin, M., Xie, Z.Z., Ge, P., Li, J., Jiang, S.S., Subburaj, S., Li, X.H., Zeller, F.J., Hsam, S.L.K., Yan, Y.M. 2012. Identification and molecular characterisation of HMW glutenin subunit 1By16* in wild emmer. J. Appl. Genet. 53:249-258.

Khatkar, B.S., Fido, R.J., Tatham, A.S., Schofield, J.D. 2002a. Functional properties of wheat gliadins. II. Effects on dynamic rheological properties of wheat gluten. J. Cereal Sci. 35:307-313.

Khatkar, B.S., Fido, R.J., Tatham, A.S., Schofield, J.D. 2002b. Functional properties of wheat gliadins. I. Effects on mixing characteristics and bread making quality. J. Cereal Sci. 35:299-306.

Li, W. 2009. Application alveograph to research quality evaluation of wheat. Heilongjiang Agri. Sci. 6:117-118. (in Chinese)

Liu, L., Ikeda, T.M., Branlard, G., Peña, R.J., Rogers, W.J., Lerner, E.S., Kolman, M.A., Xia, X.C., Wang, L.H., Ma, W.J., Appels, R., Yoshida, H., Wang, A.L., Yan, Y.M., He, Z.H. 2010. Comparison of low molecular weight glutenin subunits identified by SDS-PAGE, 2-DE, MALDI-TOF-MS and PCR in common wheat. BMC Plant Biol. 10:124.

Liu, Y.N., He, Z.H., Appels, R., Xia, X.C. 2012. Functional markers in wheat: Current status and future prospects. Theor. Appl. Genet. 125:1-10.

Lv, X.B., Zhang, Y.B., Song, Q.J., Liu, D.N., Zhang, C.L., Zhao, H.B. 2004. Qualitative difference between HMW-GS $5+10$ and 2+12 NILs of four spring wheat cultivars with high-quality genetic background. Agri. Sci. in China 3:568-574.

Melnikova, N.V., Kudryavtseva, A.V., Kudryavtsev, A.M. 2012. Catalogue of alleles of gliadin-coding loci in durum wheat (Triticum durum Desf.). Biochimie 94:551-557.

Metakovsky, E.V. 1991. Gliadin allele identification in commom wheat: II. Catalogue of gliadin alleles in common wheat. J. Genet. and Breed. 45:325-344.

Nieto-Taladriz, M.T., Perretant, M.R., Rousset, M. 1994. Effect of gliadins and HMW and LMW subunits of glutenin on dough properties in the $\mathrm{F}_{6}$ recombinant inbred lines from a bread wheat cross. Theor. Appl. Genet. 88:81-88.

Payne, P.I. 1987. Genetics of wheat storage proteins and the effect of allelic variation on bread-making quality. Ann. Rev. Plant Physiol. 38:141-153.

Payne, P.I., Jackson, E.A., Holt, L.M. 1984. The association between gamma-gliadin 45 and gluten strength in durum wheat varieties: A direct causal effect of the result or genetic linkage. J. Cereal Sci. 2:73-81.

Payne, P.I., Lawrence, G.J. 1983. Catalogue of alleles for the complex gene loci, Glu-A1, Glu-B1, and Glu-D1 which code for high-molecular-weight subunits of glutenin in hexaploid wheat. Cereal Res. Commun. 11:29-35.

Popineau, Y., Cornec, M., Lefebvre, J., Marchylo, B. 1994. Influence of high Mr glutenin subunits on glutenin polymers and rheological properties of glutens and gluten subfractions of near-isogenic lines of wheat Sicco. J. Cereal Sci. 19:231-241.

Ragupathy, R., Naeem, H.A, Reimer, E., Lukow, O.M., Sapirstein, H.D., Cloutier, S. 2008. Evolutionary origin of the segmental duplication encompassing the wheat $G L U-B 1$ locus encoding the overexpressed $\mathrm{Bx} 7$ (Bx7OE) high molecular weight glutenin subunit. Theor. Appl. Genet. 116:283-296.

Cereal Research Communications 43, 2015 
Rasheed, A., Mahmood, T., Kazi, A.G., Ghafoor, A., Mujeeb-Kazi, A. 2012. Allelic variation and composition of HMW-GS in advanced lines derived from d-genome synthetic hexaploid/bread wheat (Triticum aestivum L.). J. Crop Sci. Biotech. 15:1-7.

Redaelli, R., Morel, M.H., Autran, J.C., Pogna, N.E. 1995. Genetic analysis of low Mr glutenin subunits fractionated by two-dimensional electrophoresis (A-PAGEx SDS-PAGE). J. Cereal Sci. 21:5-13.

Redaelli, R., Pogna, N.E., Ng, P.K.W. 1997. Effects of prolamins encoded by chromosomes 1B and 1D on the rheological properties of dough in near-isogenic lines of bread wheat. Cereal Chem. 74:102-107.

Singh, N.K., Shepherd, K.W. 1988. Linkage mapping of genes controlling endosperm storage proteins in wheat. Theor. Appl. Genet. 75:628-641.

Song, W.F., Zhao, H.B., Zhang, Y.B., Li, J.L., Jiang, H.H., Song, Q.J., Zhang, C.L., Xin, W.L., Xiao, Z.M. 2012. LMW-GS identification at Gli-A1/GluA3 loci in NILs of wheat variety Longmai 19. Heilongjiang Agri. Sci. 2:1-5. (in Chinese)

Sontag-Strohm, T., Payne, P.I., Salovaara, H. 1996. Effect of allelic variation of glutenin subunits and gliadins on baking quality in the progeny of two biotypes of bread wheat cv. Ulla. J. Cereal Sci. 24:115-124.

Sozinov, A.A., Poperelya, F.A. 1970. On the inheritance of some alcohol-soluble protein fractions in hybrids of wheat. Nauchno-Tekhnich Bulleten VSGI. 13:1-4.

Sozinov, A.A., Poperelya, F.A. 1980. Genetic classification of prolamines and its use for plant breeding. Ann. Technol. Agric. 29:229-245.

Sozinov, A.A., Poperelya, F.A. 1982. Polymorphism of prolamins and variability of grain quality. Plant Food Hum. Nutr. 31:243-249.

Wang, L.H., Li, G.Y., Pena, R.J., Xia, X.C., He, Z.H. 2010. Development of STS markers and establishment of multiplex PCR for Glu-A3 alleles in common wheat (Triticum aestivum L.). J. Cereal Sci. 51:305-312.

Wei, R. 1998. A comparative study on rheological analysers for dough. J. Chin. Cereals and Oils Assoc. 13:10-12. (in Chinese)

Zhang, L.L., Zhang, Y.B., Zhao, H.B., Song, Q.J., Yu, H.Y., Zhang, C.L., Xin, W.L., Xiao, Z.M. 2008. Study on the quality of NILs of wheat cv. Longfumai 3 possessing HMW-GS Null and 1 subunits. Agri. Sci. in China 7:140-147.

Zhang, L.L., Zhang, Y.B., Li, J.L., Zhao, H.B., Song, Q.J., Yu, H.Y., Zhang, C.L., Xin, W.L., Xiao, Z.M. 2010. Quality differences between NILs of wheat variety Long 97-586 possessing HMW-GS 7+8 and 7. Sci. China Ser. C-Life Sci. 53:286-291.

Zhang, Q.C., Shao, L.G., Wang, Y., Li, C.H., Ma, Y., Che, J.Y., Gao, F.M. 2006. Analysis and evaluation of spring wheat quality with alveograph NG consistograph. Heilongjiang Agri. Sci. 5:74-77. (in Chinese)

Zhang, X.F., Liu, D.C., Yang, W.L., Liu, K.F., Sun, J.Z., Guo, X.L., Li, Y.W., Wang, D.W., Ling, H.Q., Zhang, A.M. 2011. Development of a new marker system for identifying the complex members of the low-molecular-weight glutenin subunit gene family in bread wheat (Triticum aestivum L.). Theor. Appl. Genet. 122:1503-1516.

Zhang, X.F., Jin, H., Zhang, Y., Liu, D.C., Li, G.Y., Xia, X.C., He, Z.H., Zhang, A.M. 2012. Composition and functional analysis of low-molecular-weight glutenin alleles with Aroona near-isogenic lines of bread wheat. BMC Plant Biol. 12:243.

Zhang, Y.B., Qi, S.Y., Xiao, Z.M., Xin, W.L., Gao, Z. 1997. Study of a noncontinuous formaic acid-PAGE method of wheat gliadin. Nat. Sci. J. Harbin Norm. Univ. 13:70-73. (in Chinese) 


\title{
Electronic Supplementary Material (ESM)
}

Electronic Supplementary Material (ESM) associated with this article can be found at the website of CRC at http://www.akademiai.com/content/120427/

\author{
Electronic Supplementary Table S1. Quality parameters of the three NILs of Longmai 19 \\ grown for 2 years \\ Electronic Supplementary Figure S1. SDS-PAGE banding patterns. 1: Marquis; 2: L-19-613; \\ 3: L-19-626; 4: L-19 \\ Electronic Supplementary Figure S2. A-PAGE banding patterns. 1: L-19; 2: Marquis; \\ 3: L-19-613; 4: L-19-626
}

